\title{
In vivo analysis of Saccharomyces cerevisiae plasma membrane ATPase Pma1p isoforms with increased in vitro $\mathrm{H}^{+}$/ATP stoichiometry
}

\author{
Stefan de Kok • Duygu Yilmaz • \\ Jean-Marc Daran · Jack T. Pronk • \\ Antonius J. A. van Maris
}

Received: 19 January 2012/Accepted: 22 March 2012/Published online: 10 April 2012

(C) The Author(s) 2012. This article is published with open access at Springerlink.com

\begin{abstract}
Plasma membrane $\mathrm{H}^{+}$-ATPase isoforms with increased $\mathrm{H}^{+}$/ATP ratios represent a desirable asset in yeast metabolic engineering. In vivo proton coupling of two previously reported Pmalp isoforms (Ser800Ala, Glu803Gln) with increased in vitro $\mathrm{H}^{+}$/ ATP stoichiometries was analysed by measuring biomass yields of anaerobic maltose-limited chemostat cultures expressing only the different PMAI alleles. In vivo $\mathrm{H}^{+}$/ATP stoichiometries of wildtype Pma1p and the two isoforms did not differ significantly.
\end{abstract}

Keywords Pma1 - Ser800Ala - Glu803Gln ·

Maltose $\cdot$ Yeast $\cdot$ Proton symport

\section{Introduction}

Plasma membrane $\mathrm{H}^{+}$-ATPases are ubiquitous enzymes that play an important role in eukaryotic physiology by using the free energy from ATP hydrolysis to pump protons from the cytosol, across the plasma membrane and out of the cell. In this

S. de Kok · D. Yilmaz · J.-M. Daran .

J. T. Pronk - A. J. A. van Maris ( $\square)$

Department of Biotechnology, Delft University

of Technology and Kluyver Centre for Genomics

of Industrial Fermentation, Julianalaan 67,

2628 BC Delft, The Netherlands

e-mail: A.J.A.vanMaris@TUDelft.nl way, cells maintain intracellular $\mathrm{pH}$ homeostasis and generate a proton motive force (PMF), which can be used to drive many crucial transport processes (Serrano 1991; Burgstaller 1997; Van Maris et al. 2004). Saccharomyces cerevisiae contains two distinct plasma membrane $\mathrm{H}^{+}$-ATPases encoded by the essential gene PMAI and the non-essential gene PMA2 (Serrano et al. 1986; Schlesser et al. 1988). The well-characterised plasma membrane $\mathrm{H}^{+}$-ATPase of S. cerevisiae (Serrano 1989; Morsomme et al. 2000; Morth et al. 2010) expels one proton per ATP molecule hydrolysed (Van Leeuwen et al. 1992; Weusthuis et al. 1993), even though the Gibbs free-energy of ATP hydrolysis (around $-45 \mathrm{~kJ} \mathrm{~mol}^{-1}$ under physiological conditions (Canelas et al. 2011)) should be sufficient to drive export of 2 protons $\left(+19 \mathrm{~kJ} \mathrm{~mol} \mathrm{protons}^{-1}\right.$ at a PMF of $-200 \mathrm{mV}$ (Serrano 1991)). The $\mathrm{H}^{+} / \mathrm{ATP}$ stoichiometry of the plasma membrane $\mathrm{H}^{+}$-ATPase determines the ATP requirement for cellular homeostasis and maintenance of the PMF. Moreover, it influences the biomass yield on substrates whose import makes use of the PMF (e.g., maltose and $\mathrm{NH}_{4}{ }^{+}$) (Van Leeuwen et al. 1992; Weusthuis et al. 1993; Marini et al. 1997), it can influence tolerance to both low $\mathrm{pH}$ and weak organic acids (Verduyn et al. 1992; Piper et al. 1998; Abbott et al. 2007) and it can have a crucial impact on the stoichiometry and kinetics of organic acid production by engineered strains of $S$. cerevisiae (Van Maris et al. 2004; Sauer et al. 2008; Abbott et al. 2009). Increasing the $\mathrm{H}^{+} / \mathrm{ATP}$ stoichiometry of the 
S. cerevisiae plasma membrane $\mathrm{H}^{+}$-ATPase could therefore present many interesting opportunities for metabolic engineering.

Two isoforms of the S. cerevisiae plasma membrane $\mathrm{H}^{+}$-ATPase Pmalp have been described that displayed an increased in vitro $\mathrm{H}^{+} /$ATP stoichiometry: Pmalp ${ }^{\text {Ser800Ala }}$ (Guerra et al. 2007) and Pma1 $\mathrm{p}^{\text {Glu803Gln }}$ (Petrov et al. 2000). However, no improved tolerance to low $\mathrm{pH}$ was observed after introducing the Glu803Gln mutation in PMA1 (Guerra et al. 2007). The present study investigates whether the in vivo $\mathrm{H}^{+}$/ ATP stoichiometry of $S$. cerevisiae Pma1p isoforms can be analysed via the anaerobic biomass yield on maltose of engineered strains. In S. cerevisiae maltose is imported via a proton-symport mechanism (Van Leeuwen et al. 1992). Due to subsequent proton export by the plasma membrane $\mathrm{H}^{+}$-ATPase at a stoichiometry of $1 \mathrm{H}^{+}$/ATP, conversion of the disaccharide maltose to ethanol only yields 3 ATP (1.5 ATP per hexose equivalent) (Van Leeuwen et al. 1992; Weusthuis et al. 1993). As a result, the anaerobic biomass yield on maltose is $25 \%$ lower per hexose equivalent than the anaerobic biomass yield on glucose (2 ATP per hexose) (Van Leeuwen et al. 1992; Weusthuis et al. 1993). In theory, an increased stoichiometry of the plasma membrane $\mathrm{H}^{+}$-ATPase will increase the biomass yield on maltose due to a decreased energy requirement of all processes that require proton extrusion (e.g., maintenance and generation of the PMF and import of maltose and $\mathrm{NH}_{4}{ }^{+}$). Even when only the lower ATP-requirement for maltose import is taken into account (Weusthuis et al. 1993), a stoichiometry of $2 \mathrm{H}^{+} /$ATP is already expected to result in a $17 \%$ increase of the biomass yield on maltose. To characterise the in vivo $\mathrm{H}^{+} / \mathrm{ATP}$ stoichiometry of the Ser800Ala and Glu803Gln isoforms of Pma1p, the wild-type PMAI allele was replaced by the corresponding $P M A 1$ mutant alleles in a pma $2 \Delta$ background and the anaerobic biomass yields on maltose were compared to those of an isogenic PMA1 pma2A reference strain.

\section{Introduction of Ser800Ala and Glu803GIn mutations in PMA1}

To introduce the Ser800Ala (TCC $\rightarrow$ GCT) and Glu803Gln (GAA $\rightarrow$ CAA) mutations into PMA1, DNA from the KpnI site in PMAI until the NgoMIV site in LEU1 was amplified from CEN.PK113-7D genomic DNA using primers PMA1 Fw and LEU1 Rv (for primers, see Table 1) and cloned into pENTR/DTOPO using Gateway technology (Invitrogen, Carlsbad, USA), resulting in pUD109 (for plasmids, see Table 2). To introduce extra restriction sites in the intergenic region between $P M A I$ and $L E U 1$, DNA was
Table 1 Primers used in this study

\begin{tabular}{ll}
\hline Primer name & Sequence $\left(5^{\prime} \rightarrow 3^{\prime}\right)$ \\
\hline PMA1 Fw & CACCGGGTACCAACATTTACAACGCTG \\
LEU1 Rv & CAACTCTTCTGACCTTTCTGCC \\
LEU1p Fw & CTCTAGACACTAGTATGCCGTACGTGACTCA \\
& GTTTAGTCTGACCTTC \\
LEU1p Rv & CCTTCGAAAGCTTGTGGAG \\
PMA1 Ctrl Fw & GGATCCACCAAGAGACGATACTG \\
LEU1 Ctrl Rv & CCGAAATATGGAACGCCGAACTG \\
hphN1 Fw & ACGCGGATTTCGGCTCCAAC \\
hphNT1 Rv & AGACGTCGCGGTGAGTTCAG \\
PMA2 KO Fw & TCGTTGCTGTGTGCTAGTACAATTTAAGCAAAAGG \\
PMA2 KO Rv & AAACTGTTTTGCGTTCAGCTGAAGCTTCGTACGC \\
PMA2 Ctrl Fw & CTTGGTATCGACAAATTGAAATGAAAATGAGGAATAA \\
PMA2 Ctrl Rv & CAAAAAGGAGATCGCATAGGCCACTAGTGGATCTG \\
KanMX Fw & GGCGGTGTGATGGTACTTC \\
KanMX Rv & CGGCCTACTTCTGATATGTGG \\
\hline
\end{tabular}


Table 2 Plasmids used in this study

\begin{tabular}{|c|c|c|}
\hline Plasmid & Characteristic & Reference/source \\
\hline pENTR/D-TOPO & Gateway entry clone & Invitrogen, USA \\
\hline pUG6 & $\begin{array}{l}\text { PCR template for loxP-KanMX4- } \\
\text { loxP cassette }\end{array}$ & (Gueldener et al. 2002) \\
\hline pSH65 & $\begin{array}{l}\text { Centromeric plasmid, ble }{ }^{R} \\
\mathrm{P}_{G A L 1} \text { Cre- } \mathrm{T}_{C Y C l}\end{array}$ & (Gueldener et al. 2002) \\
\hline pUG-hphNT1 & $\begin{array}{l}\text { PCR template for loxP-hphNT1- } \\
\quad \text { loxP cassette }\end{array}$ & (De Kok et al. 2011) \\
\hline pUD117 & $\begin{array}{l}\text { pUC57, synthetic construct } \\
\text { 'PMA1 }\end{array}$ & $\begin{array}{l}\text { Baseclear BV, } \\
\text { The Netherlands }\end{array}$ \\
\hline pUD118 & $\begin{array}{l}\text { pUC57, synthetic construct } \\
\quad \text { 'PMA1 }\end{array}$ & $\begin{array}{l}\text { Baseclear BV, } \\
\text { The Netherlands }\end{array}$ \\
\hline pUD109 & $\begin{array}{l}\text { Gateway entry clone, } \\
\text { 'PMAl-LEU1' }\end{array}$ & This study \\
\hline pUD113 & $\begin{array}{l}\text { Gateway entry clone, 'PMA1-multiple } \\
\text { cloning site-LEU1' }\end{array}$ & This study \\
\hline pUD119 & $\begin{array}{l}\text { Gateway entry clone, 'PMA1 } 1^{S 800 A} \text {-multiple } \\
\text { cloning site-LEU1' }\end{array}$ & This study \\
\hline pUD120 & $\begin{array}{l}\text { Gateway entry clone, 'PMA1 } 1^{E 803 Q} \text {-multiple } \\
\text { cloning site-LEU1' }\end{array}$ & This study \\
\hline pUD124 & 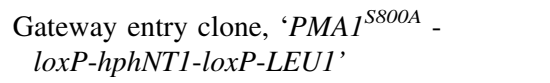 & This study \\
\hline pUD125 & $\begin{array}{l}\text { Gateway entry clone, 'PMA1 }{ }^{E 803 Q} \text { - } \\
\text { loxP-hphNT1-loxP-LEU1' }\end{array}$ & This study \\
\hline
\end{tabular}

amplified from pUD109 using primers LEU1p Fw and LEU1p Rv. The resulting PCR product was restricted with $X b a \mathrm{I}$ and HindIII and ligated into pUD109, resulting in pUD113. To introduce point mutations in PMAl, the KpnI-SalI fragment of pUD117 and pUD118, containing synthesized parts of PMA1 including the Ser800Ala (TCC $\rightarrow$ GCT) and Glu803Gln (GAA $\rightarrow$ CAA) mutations, were ligated into pUD113, resulting in pUD119 and pUD120 (Table 2). To introduce the hygromycin B resistance marker hphNT1, a SpeI-BsiWI fragment of pUG-hphNTl was ligated into pUD119 and pUD120, resulting in pUD124 and pUD125, respectively. The KpnI-NgoMIV fragment of pUD124 and pUD125 was transformed to CEN.PK1137D resulting in IMI058 and IMI059, respectively (for strains, see Table 3). Correct integration of the cassette was confirmed via PCR using primer pairs PMA1 Ctrl Fw/hphNT1 Rv and hphNT1 Fw/LEU1 Ctrl Rv. To remove the hygromycin $\mathrm{B}$ resistance marker gene $h p h N T 1$, IMI058 and IMI059 were transformed with pSH65 and-after marker removal via the Cre/loxP system (Gueldener et al. 2002) and curing of the pSH65 plasmid-designated IMI062 and IMI063, respectively. To knockout PMA2, a cassette was amplified from
pUG6 using primers PMA2 KO Fw and PMA2 KO Rv and transformed into CEN.PK113-7D, IMI062 and IMI063, resulting in IMK328, IMX051B and IMX052, respectively. Correct knockout was confirmed via PCR using the primer pairs PMA2 Ctrl Fw/KanMX Rv and KanMX Fw/PMA2 Ctrl Rv. Presence of the introduced point mutations was verified by duplicate amplification of PMA1 using primers PMA1 Ctrl Fw and LEU1 Ctrl $\mathrm{Rv}$ and sequencing approximately $200 \mathrm{bp}$ up- and downstream of the introduced mutations (Baseclear BV, Leiden, The Netherlands). Strain maintenance, yeast transformations and molecular biology techniques were performed as described previously (De Kok et al. 2011).

\section{Analysis of the in vivo stoichiometry of Pma1p ${ }^{\text {Ser800Ala }}$ and Pma1p ${ }^{\text {Glu803Gln }}$}

To analyse in vivo $\mathrm{H}^{+}$/ATP stoichiometry of the Pma1p isoforms, anaerobic chemostat experiments with maltose as the sole carbon source were performed at pH 5.0 as described previously (De Kok et al. 2011). To prevent evolutionary adaptation, the cultures were sampled within 12 volume changes. In agreement with 
Table 3 Saccharomyces cerevisiae strains used in this study

\begin{tabular}{|c|c|c|}
\hline Strain & Relevant genotype & Reference \\
\hline CEN.PK113-7D & MATa PMA1 PMA2 & $\begin{array}{l}\text { (Van Dijken et al. 2000, } \\
\text { Entian and Kotter 2007) }\end{array}$ \\
\hline IMK328 & MATа PMA1 pma2::loxP-KanMX4-loxP & This study \\
\hline IMI058 & MATa $P M A 1^{\text {S800A }}$-loxP-hphNT1-loxP PMA2 & This study \\
\hline IMI059 & MATa $P M A 1^{E 803 Q}$-loxP-hphNT1-loxP PMA2 & This study \\
\hline IMI062 & MATa $P M A 1^{S 800 A} P M A 2$ & This study \\
\hline IMI063 & MATa $P M A 1^{E 803 Q} P M A 2$ & This study \\
\hline IMX051B & MATa $P M A 1^{\text {S800A }}$ pma2::loxP-KanMX4-loxP & This study \\
\hline IMX052 & MATа $P M A 1^{E 803 Q}$ pma2::loxP-KanMX4-loxP & This study \\
\hline
\end{tabular}

model predictions based on a $\mathrm{H}^{+} /$ATP stoichiometry of 1.0 and previous observations under the same experimental conditions, the anaerobic biomass yield on maltose of the reference strain CEN.PK113-7D (PMA1 PMA2) was $24 \pm 0 \%$ lower per hexose equivalent than the anaerobic biomass yield on glucose (Table 4). The biomass yield of the engineered strains IMX051B (PMA1 ${ }^{\text {Ser800Ala }}$ pma2A) and IMX052 (PMA1 ${ }^{\text {Glu803Gln }}$ pma2 $\Delta$ ) was not higher than the yield of the reference strain CEN.PK113-7D (PMA1 PMA2) or the isogenic strain IMK328 (PMA1 pma2 $\Delta$ ) (Table 4). At the end of the chemostat experiments, genomic DNA was extracted and used for duplicate amplification of part of PMA1. Subsequent sequencing confirmed that the introduced mutations were still present. Apparently, the Ser800Ala and Glu803Gln mutations in PMA1 did not increase the in vivo $\mathrm{H}^{+}$/ATP stoichiometry under the tested conditions, in contrast to what has been reported previously using in vitro assays (Petrov et al. 2000; Guerra et al. 2007). To test whether these contradictory results were due to differences in $\mathrm{pH}$ used in the in vivo $(\mathrm{pH}$ 5.0) and in vitro $(\mathrm{pH}$ 6.7) experiments, the chemostat experiments were repeated at $\mathrm{pH}$ 6.7. Also under these conditions, the difference in anaerobic biomass yield on glucose and maltose of the reference strain CEN.PK113-7D (PMA1 PMA2) at pH 6.7 was $24 \pm 0 \%$ (Table 4). Interestingly, at pH 6.7 deletion of PMA2 seemed to increase the biomass yield on maltose by $5.4 \pm 0.0 \%$ when comparing the reference strain CEN.PK113-7D (PMA1 PMA2) and IMK328 (PMA1 pma2A). However, the biomass yields on maltose of the engineered strains IMX051B (PMA1 ${ }^{\text {Ser800Ala }}$ pma2 4$)$ and IMX052 (PMA1 Glu803Gln pma2 $\Delta)$ were identical to the isogenic reference strain IMK328 (PMA1 pma2 $\Delta$ ) (Table 4). Thus, at both $\mathrm{pH}$ 5.0 and $\mathrm{pH} 6.7$ introduction of the Ser800Ala and Glu803Gln isoforms in Pmalp did not increase the in vivo $\mathrm{H}^{+} /$ATP stoichiometry.

In vitro studies are an essential tool in gaining increased understanding of membrane proteins such as $\mathrm{H}^{+}$-ATPase (Serrano 1989; Morsomme et al. 2000;

Table 4 Anaerobic biomass yields of Saccharomyces cerevisiae strains CEN.PK113-7D (PMA1 PMA2), IMK328 (PMA1 pma2 $)$, IMX051B (PMA1 ${ }^{\text {Ser80oAla }}$ pma2A) and IMX052

(PMA1 ${ }^{\text {Glu803Gln }}$ pma2 $\Delta$ ) in anaerobic sugar-limited chemostat cultures at a dilution rate of $0.10 \mathrm{~h}^{-1}$. Averages and mean deviations were obtained from duplicate cultures

\begin{tabular}{|c|c|c|c|c|}
\hline \multirow[t]{2}{*}{ Strain } & \multirow{2}{*}{$\begin{array}{l}\text { Relevant } \\
\text { genotype }\end{array}$} & \multirow{2}{*}{$\begin{array}{l}\text { Carbon } \\
\text { source }\end{array}$} & \multicolumn{2}{|c|}{ Biomass yield ( $\mathrm{g}$ g gluc $\mathrm{eq}^{-1}$ ) } \\
\hline & & & pH 5.0 & pH 6.7 \\
\hline CEN.PK113-7D & PMA1 PMA2 & Glucose & $0.095 \pm 0.002$ & $0.087 \pm 0.001$ \\
\hline CEN.PK113-7D & PMA1 PMA2 & Maltose & $0.072 \pm 0.000$ & $0.066 \pm 0.000$ \\
\hline IMK328 & PMA1 pma2 $\Delta$ & Maltose & $0.072 \pm 0.001$ & $0.070 \pm 0.000$ \\
\hline IMX051B & $P M A 1^{\text {Ser800Ala }}$ pma $2 \Delta$ & Maltose & $0.073 \pm 0.001$ & $0.069 \pm 0.002$ \\
\hline IMX052 & PMA1 $1^{\text {Glu803Gln }}$ pma2 $\Delta$ & Maltose & $0.073 \pm 0.001$ & $0.069 \pm 0.001$ \\
\hline
\end{tabular}


Morth et al. 2010). Several factors may explain why the Ser800Ala and Glu803Gln isoforms of Pma1p $\mathrm{H}^{+}$-ATPase isoforms, which were clearly shown to translocate 2-3 protons per ATP in vitro (Petrov et al. 2000; Guerra et al. 2007), did not lead to a significantly increased in vivo $\mathrm{H}^{+}$/ATP stoichiometry in the anaerobic, maltose-limited cultures. Even when in vitro studies attempt to mimic in vivo conditions (e.g., $\mathrm{pH}$ and osmolarity), subtle differences in membrane composition between the plasma membrane and secretory vesicle membrane (Van der Rest et al. 1995) might affect the three-dimensional structure and functioning of the plasma membrane $\mathrm{H}^{+}$-ATPase. Additionally, thermodynamics of the proton-motive force and/or ATP hydrolysis may be different under in vitro and in vivo conditions. If the PMF in the secretory vesicles, which has not been measured (Petrov et al. 2000; Guerra et al. 2007), is significantly lower than the in vivo PMF, this would make an increased $\mathrm{H}^{+}$/ATP stoichiometry thermodynamically easier to achieve in vitro, but not in vivo. This difference between the in vitro and in vivo thermodynamic potential of the $\mathrm{H}^{+}$-ATPase becomes even more striking for the free energy of ATP hydrolysis. In the in vitro assays, ADP and inorganic phosphate were not added to the reaction mixture and only ATP was added from the start. Especially during the early stages of the assay, which coincides with the determination of the $\mathrm{H}^{+}$/ATP stoichiometry, this created a non-physiologically high driving force for ATP hydrolysis, which will drastically exceed the estimated $-45 \mathrm{~kJ} \mathrm{~mol}^{-1}$ under physiological conditions (Canelas et al. 2011). Analogously, due to cellular homeostasis and fluxversus-stoichiometry constraints techniques such as membrane potential determination or extracellular acidification measurements do not allow accurate in vivo analysis of the $\mathrm{H}^{+}$/ATP stoichiometry. Therefore, the method presented in this study, in which in vivo proton coupling of plasma membrane $\mathrm{H}^{+}$-ATPase isoforms was analysed via its impact on the biomass yields of anaerobic, maltose-grown cultures, provides a useful tool in the continuing search for Pmalp isoforms and/or heterologous plasma membrane $\mathrm{H}^{+}$-ATPases with an in vivo $\mathrm{H}^{+}$/ATP ratio above 1.0 in growing yeast cultures.

Acknowledgments This work was financially supported by Tate \& Lyle Ingredients Americas Inc. The Kluyver Centre for
Genomics of Industrial Fermentations is supported by The Netherlands Genomics Initiative.

Open Access This article is distributed under the terms of the Creative Commons Attribution License which permits any use, distribution, and reproduction in any medium, provided the original author(s) and the source are credited.

\section{References}

Abbott DA, Knijnenburg TA, de Poorter LMI, Reinders MJT, Pronk JT, van Maris AJA (2007) Generic and specific transcriptional responses to different weak organic acids in anaerobic chemostat cultures of Saccharomyces cerevisiae. FEMS Yeast Res 7:819-833

Abbott DA, Zelle RM, Pronk JT, van Maris AJA (2009) Metabolic engineering of Saccharomyces cerevisiae for production of carboxylic acids: current status and challenges. FEMS Yeast Res 9:1123-1136

Burgstaller W (1997) Transport of small ions and molecules through the plasma membrane of filamentous fungi. Crit Rev Microbiol 23:1-46

Canelas AB, Ras C, ten Pierick A, van Gulik WM, Heijnen JJ (2011) An in vivo data-driven framework for classification and quantification of enzyme kinetics and determination of apparent thermodynamic data. Metab Eng 13:294-306

De Kok S, Yilmaz Y, Suir E, Pronk JT, Daran JM, van Maris AJA (2011) Increasing free-energy (ATP) conservation in maltose-grown Saccharomyces cerevisiae by expression of a heterologous maltose phosphorylase. Metab Eng 13: $518-526$

Entian K, Kotter P (2007) Yeast genetic strain and plasmid collections. In: Stansfield I, Stark M.J.R (eds) Yeast gene analysis. Academic Press, San Diego. Methods Microbiol 36: 629-666

Gueldener U, Heinisch J, Koehler GJ, Voss D, Hegemann JH (2002) A second set of loxP marker cassettes for Cremediated multiple gene knockouts in budding yeast. Nucleic Acids Res 30:e23

Guerra G, Petrov VV, Allen KE, Miranda M, Pardo JP, Slayman CW (2007) Role of transmembrane segment M8 in the biogenesis and function of yeast plasma-membrane $\mathrm{H}^{+}$-ATPase. Biochim Biophys Acta Biomembr 1768:2383-2392

Marini AM, Soussi-Boudekou S, Vissers S, Andre B (1997) A family of ammonium transporters in Saccharomyces cerevisiae. Mol Cell Biol 17:4282-4293

Morsomme P, Slayman CW, Goffeau A (2000) Mutagenic study of the structure, function and biogenesis of the yeast plasma membrane $\mathrm{H}^{+}$-ATPase. Biochim Biophys Acta 1469: 133-157

Morth JP, Pedersen BP, Buch-Pedersen MJ, Andersen JP, Vilsen B, Palmgren MG, Nissen P (2010) A structural overview of the plasma membrane $\mathrm{Na}^{+}, \mathrm{K}^{+}$-ATPase and $\mathrm{H}^{+}$-ATPase ion pumps. Nat Rev Mol Cell Biol 12:60-70

Petrov VV, Padmanabha KP, Nakamoto RK, Allen KE, Slayman CW (2000) Functional role of charged residues in the 
transmembrane segments of the yeast plasma membrane $\mathrm{H}^{+}$-ATPase. J Biol Chem 275:15709-15716

Piper P, Mahé Y, Thompson S, Pandjaitan R, Holyoak C, Egner R, Mühlbauer M, Coote P, Kuchler K (1998) The Pdr12 $\mathrm{ABC}$ transporter is required for the development of weak organic acid resistance in yeast. EMBO J 17:4257-4265

Sauer M, Porro D, Mattanovich D, Branduardi P (2008) Microbial production of organic acids: expanding the markets. Trends Biotechnol 26:100-108

Schlesser A, Ulaszewski S, Ghislain M, Goffeau A (1988) A second transport ATPase gene in Saccharomyces cerevisiae. J Biol Chem 263:19480-19487

Serrano R (1989) Structure and function of plasma membrane ATPase. Annu Rev Plant Biol 40:61-94

Serrano R (1991) Transport across yeast vacuolar and plasma membranes. Cold Spring Harbor Monograph Archive 21: 523-585

Serrano R, Kielland-Brandt M, Fink G (1986) Yeast plasma membrane ATPase is essential for growth and has homology with $\left(\mathrm{Na}^{+}+\mathrm{K}^{+}\right), \mathrm{K}^{+}$- and $\mathrm{Ca}^{2+}$-ATPases. Nature 319:689-693

Van der Rest M, Kamminga AH, Nakano A, Anraku Y, Poolman B, Konings WN (1995) The plasma membrane of
Saccharomyces cerevisiae: structure, function, and biogenesis. Microbiol Mol Biol Rev 59:304-322

Van Dijken JP, Bauer J, Brambilla L, Duboc P, Francois JM, Gancedo C, Giuseppin MLF, Heijnen JJ, Hoare M, Lange $\mathrm{HC}$ (2000) An interlaboratory comparison of physiological and genetic properties of four Saccharomyces cerevisiae strains. Enzyme Microb Technol 26:706-714

Van Leeuwen CC, Weusthuis RA, Postma E, van den Broek PJ, van Dijken JP (1992) Maltose/proton co-transport in Saccharomyces cerevisiae. Comparative study with cells and plasma membrane vesicles. Biochem J 284:441-445

Van Maris AJA, Konings WN, van Dijken JP, Pronk JT (2004) Microbial export of lactic and 3-hydroxypropanoic acid: implications for industrial fermentation processes. Metab Eng 6:245-255

Verduyn C, Postma E, Scheffers WA, van Dijken JP (1992) Effect of benzoic acid on metabolic fluxes in yeasts: a continuous culture study on the regulation of respiration and alcoholic fermentation. Yeast 8:501-517

Weusthuis RA, Adams H, Scheffers WA, van Dijken JP (1993) Energetics and kinetics of maltose transport in Saccharomyces cerevisiae: a continuous culture study. Appl Environ Microbiol 59:3102-3109 